Title

\title{
Correlation between hemoglobin, serum albumin, body mass index, hemodialysis shift time and hemodialysis adequacy with quality of life in hemodialysis patients
}

\begin{abstract}
Authors:
Darryl Virgiawan Tanod1, Linda Rotty2, Stella Palar3, Emma Moeis 3

1Departement of Internal Medicine, Sam Ratulangi University/ Prof.Dr.R.D.Kandou Hospital Manado 2Hematology-Oncology Division, Departement of Internal Medicine, Sam Ratulangi University

Prof.Dr.R.D.Kandou Hospital Manado

3 Nephrology and Hypertension Division, Departement of Internal Medicine, Sam Ratulangi University

Prof.Dr.R.D.Kandou Hospital Manado
\end{abstract}

Editor:

Syakib Bakri

Received 18 June 2018, revised 13 September 2018, accepted 12 October 2018, published 1 December 2018

\section{Abstract}

Background CKD Patients on routine hemodialysis (HD) are prone to medical complications and conditions that are potentially detrimental to the quality of life (QoL), such as anemia, malnutrition, low body mass index (BMI), HD shift time, and $\mathrm{HD}$ adequacy measured by $\mathrm{Kt} / \mathrm{V}$. CKD patients undergoing routine HD mostly have lowered QoL and are at higher risk for malnutrition, inflammation, hospitalization, and mortality, compared to the general population. This study intends to find out whether there is a correlation between these factors and the quality of life of patients undergoing routine hemodialysis. Methods The design of this study is a cross-sectional analysis of observational data. Hemodialysis patients from general hospital Prof.dr. R. D.Kandou Manado for 3 months from August to October 2017 wereincluded. Fifty-two patients meet the inclusion and exclusion criteria. The correlation between quality of life with anemia, serum albumin, BMI, adequacy of HD, using Pearson correlation test (if normality test fulfilled) or Spearman correlation test (if the normality test not fulfilled) and Independent Samples T-test to assess the quality of life with HD shift time. Results This study found no correlation between hemoglobin levels ( $p=0.244, r=-0.098), B M I(p=0.473$, $\mathrm{r}=-0.010)$, HD timing $(\mathrm{p}=0.082)$ and quality of life of the patients, but a significant correlation between serum albumin $(\mathrm{p}=0.020, \mathrm{r}=0.286)$, HD adequacy measured by Kt/V ( $\mathrm{p}=0.030, \mathrm{r}=0.257)$ and subjects' quality of life. Conclusion This study showed that serum albumin and $\mathrm{Kt} / \mathrm{V}$ values had a significant correlation with quality of life, while hemoglobin, BMI and dialysis shift time are not related to the quality of life.

Keywords quality of life, hemoglobin, BMI, albumin, hemodialysis adequacy

\section{Corresponding Author}

e-mail: darrylvirgiawan@gmail.com (Tanod, D)

phone : +628114340811

\section{BACKGROUND}

Chronic kidney disease (CKD) is a pathological process with a multitude of underlying etiologies, marked by the progressive decline of renal function of chronic and irreversible nature, and generally ends up with end-stage renal disease (ESRD) requiring renal replacement therapy (RRT) such as dialysis or kidney transplantation. ${ }^{1}$

Hemodialysis is still the primary modality of choice for RRT compared to peritoneal dialysis and kidney transplantation. National Kidney Foundation (NKF) USA data shows over 2 million patients with CKD received hemodialysis, while approximately only 15.000 patients in Indonesia, this number of patients shows a steady incline annually. ${ }^{2}$

Patients on routine hemodialysis are prone to medical complications and conditions that are potentially detrimental to the quality of life (QoL), such as anemia, malnutrition, low body mass index (BMI), HD time and adequacy. ${ }^{2,3}$ 
Quality of life (QoL) is defined as a condition in which patients feel physically, mentally, socially, and spiritually comfortable and can lead a life for their own wellbeing and others'. CKD patients undergoing routine HD mostly have lowered QoL and are at higher risk for malnutrition, inflammation, hospitalization, and mortality compared to the general population. QoL is measured through patient's perception of their disease and used as an important clinical outcome to evaluate HD benefit. One of the modalities used to measure QoL is the Short Form Health Survey with 36 Questions (SF-36). SF-36 is a documented and validated system of evaluation for QoL, appropriate for the general population and HD patients., 3,10

Anemia has been known to be an important factor in QoL. The negative impact of anemia has been found in predialysis and dialysis patients. ${ }^{4}$ Moreno et al showed that the increase of hematocrit level nearing normal values in HD patients is related to an increase in QoL. ${ }^{5}$ A study by Beusterien et al showed patients on dialysis receiving erythropoietin (EPO) therapy have better QoL compared to those who didn't receive EPO. ${ }^{6}$

Nutrition is also an important factor affecting QoL, being related to the mortality rate in CKD patients especially those undergoing routine HD. A study from The Italian DIA-QOL group on 300 patients undergoing routine HD shows, patients with low serum albumin have lower SF-36 scores, specifically in the physical section. ${ }^{4,7,10}$ Normal BMI in ESRD patients on HD is associated with higher QoL and is also shown to have a protective effect resulting in a higher survival rate. An increase of BMI reduces $10 \%$ mortality rate in patients on routine HD. ${ }^{7,8}$

The time spent on dialysis also cause problems in QoL. The assigned schedule for morning HD has been suspected to cause a higher rate of depression in patients. The adequacy of HD has also been linked to QoL. ${ }^{2,9}$

The adequacy of HD can be measured by laboratory values formulated as urea reduction ratio (URR) and the ratio of the volume of blood dialyzed per time unit and post dialysis estimated blood volume, known as $\mathrm{Kt} / \mathrm{V}$. The Indonesian Society of Nephrology has established the ideal value for $\mathrm{Kt} / \mathrm{V}$ should be $\geq 1.8$ for patients scheduled for twice-weekly HD with 5 hours per session. ${ }^{10,11}$

\section{OBJECTIVES}

To determine the correlation between anemia, serum albumin, BMI, HD adequacy and differences in HD shift time with QOL in subjects with chronic kidney disease undergoing regular HD.

\section{METHODS}

Design of the study is a cross-sectional analysis of observational data. Patients were selected among those attending hemodialysis unit of general hospital Prof.dr. R. D. Kandou Manado from August 2017 - October 2017. Inclusion criteria were patients of 18 years of age or older, who were on routine 5-hour hemodialysis sessions twice a week for a minimum of three months, with $\mathrm{Hb}>5 \mathrm{~g} \%$, and dry weight already achieved, who are willing to participate in the study by signing informed consent letter. Dry weight is defined as weight without excess fluid that could be tolerated by the patient without any problems or hypotension and was measured at the time the patient did not experience dizziness or cramps between dialysis sessions, had no swelling in the feet, ankles, arms, hands, or around the eyes, and respiration was comfortable and easy. Exclusion criteria are patients with active bleeding and/or disturbance in consciousness. HD shift time was categorized into morning and afternoon HD shift. Quality of life was measured through the SF-36 questionnaire, with a normal score of $\geq 60$.

Patients who were the subject of the study underwent an examination process starting with anamnesis, standard physical examination, and had to answer questions in the SF-36 questionnaire. Laboratory examinations included hemoglobin, serum albumin levels, and $\mathrm{Kt} / \mathrm{V}$ value calculated using Daugirdas formula.

\section{RESULTS}

The study was conducted in HD patients Prof. dr.R.D.Kandou Manado general hospital for three months, starting from August 2017 and ended in October 2017. The subjects eligible for the study consisted of 52 patients on routine hemodialysis; the demographic variables of the subjects were tested for normal distribution using the Kolmogorov-Smirnov test. (Table 1)

Hemodialysis was done on patients in the fifth decade in 19 subjects $(36.5 \%)$, followed by the fourth decade in 14 subjects $(26.8 \%), 2$ subjects $(3.8 \%)$ in the $2^{\text {nd }}$ decade and one $(9 \%)$ in the $8^{\text {th }}$ decade. Most subjects were male 28 subjects $(53.8 \%)$ while 24 subjects (46.2\%) were women. Hb level of 8.0-9.9 gr\% (mild anemia) was found in $(50 \%)$ of subjects and no significant correlation $(p=0.244)$ was found with QOL. Albumin values in the range of 3.5-4.5 was found in as many as 37 subjects $(71.2 \%)$ and a significant correlation ( $p=0.020)$ was found with QOL, BMI value in the range of $18.5-22.9 \mathrm{Kg} / \mathrm{m}^{2}$ (normal weight) was found in 24 subjects $(46.15 \%)$ and no significant correlation $(p=0.473$ ) with QOL. Kt/V > 1,0-1,7 was found in 40 subjects $(76.92 \%)$ with a significant correlation with 
Table 1. Subjects' baseline characteristics

\begin{tabular}{lcccccc}
\hline Characteristic & N & Min & Max & Median & Mean & SD \\
\hline Age (yrs) & 52 & 27.00 & 82.00 & 51.50 & 51.23 & 11.82 \\
SF-36 & 52 & 15.30 & 95.20 & 63.53 & 63.36 & 19.46 \\
Hemoglobin (g\%) & 52 & 5.20 & 14.30 & 9.05 & 9.14 & 1.75 \\
BMI (kg/m ( $)_{\text {Serum Albumin (g/dL) }}^{52}$ & 16.88 & 30.00 & 22.68 & 22.52 & 3.13 \\
Kt/v & 52 & 1.93 & 5.70 & 4.04 & 3.89 & 0.65 \\
Duration of HD & 52 & 0.59 & 1.84 & 1.20 & 1.21 & 0.26 \\
& 52 & 0.03 & 6.06 & 1.030 & 1.42 & 1.51 \\
\hline
\end{tabular}

Table 2. Correlation of QOL with $\mathrm{Hb}$, albumin, BMI and $\mathrm{Kt} / \mathrm{V}$

QOL (SF-36)

\begin{tabular}{cccc}
\cline { 2 - 3 } Hb & $\mathrm{n}$ & $\mathrm{r}$ & $\mathrm{p}$ \\
Albumin & 52 & -0.098 & 0.244 \\
$\mathbf{B M I}$ & 52 & 0.286 & 0.020 \\
$\mathbf{K t} / \mathbf{V}$ & 52 & -0.010 & 0.473 \\
\hline
\end{tabular}

$\mathrm{QOL}=$ Quality of life, $\mathrm{Hb}=$ Hemoglobin, $\mathrm{BMI}=$ Body mass index

QOL $(\mathrm{p}=0.033)$. (Table 2)

Twenty-six subjects $(50 \%)$ had HD sessions in the morning and 26 subjects underwent $\mathrm{HD}$ session in the afternoon. To assess the difference between HD shift time and QOL, the data was initially tested with Kolmogorov-Smirnov normality test and a normal data distribution was obtained The data was then tested with the Independent Samples T-test and no significant difference was found $(\mathrm{p}=0.082)$.

\section{DISCUSSION}

Fifty-two patients consisting of more male patients (53.8\%), is consistent with previous studies such as a study by Hecking in 2013, stating that male CKD and ESRD patients are more likely to receive hemodialysis compared to female patients. ${ }^{12}$ The subjects of the study were mostly around the age of $50-59$ years $(36.5 \%)$, which is also consistent with the data from National Kidney Foundation (USA) in 2015 which stated that most CKD patients (44.3\%) were $45-64$ years old. ${ }^{13}$

The correlation between $\mathrm{Hb}$ level and subjects' QoL was not found to be significant ( $\mathrm{p}=0.244)$. Half of the subjects had a $\mathrm{Hb}$ level of $8.0-9.0 \mathrm{~g} \%$ (mild anemia), followed by $\mathrm{Hb}$ level of $10.0-13.0 \mathrm{~g} \%$ in $25 \%$ of subjects. Study by Elwood et al in 1969 showed that in 880 female patients, signs and symptoms of anemia such as palpitation, dizziness, dyspnea, and cephalgia usually developed when the $\mathrm{Hb}$ level reached 7-8 $\mathrm{g} \%{ }^{14}$ Herbert et al in 1997, through systematic review, showed that $56 \%$ of patients with anemia developed compensatory mechanism such as redistribution of coronary and cerebral blood flow. ${ }^{15}$ A study by Drueke et al in 2006 showed that no significant reduction in QoL measured with SF-36, was found in patients with $\mathrm{Hb}$ level 11-12 $\mathrm{gr} \%$ treated with immediate correction, compared to patients with $<10$ $\mathrm{g} \% .^{16}$

The correlation between QoL with serum albumin level tested using Pearson correlation test showed significance $(p=0.020)$. Most subjects $(71.2 \%)$ had albumin level in the normal range of $3.5-4.5 \mathrm{~g} /$ dL with a mean value of $3.89 \mathrm{~g} / \mathrm{dL}$. This result is consistent with the study conducted by Soleymanian et al (2017) stating a significant correlation between albumin level and QoL in 417 patients undergoing routine hemodialysis. ${ }^{17}$ The study used SF-36 to measure QoL and concluded that the increase of albumin level is related to the increase of QoL. ${ }^{17}$ Soleymanian et al also found that a decreased mortality rate was found with albumin level > $3.60-$ 
$3.85 \mathrm{~g} / \mathrm{dL} .{ }^{17}$ Kubrusly et al classified patients on HD an albumin level of $<3.2 \mathrm{~g} / \mathrm{dL}$ as malnourished and level of $>3.7 \mathrm{~g} / \mathrm{dL}$ as normal. ${ }^{18}$ A study by Kalantar-Zadeh et al showed subjects with high serum albumin level had higher QoL. ${ }^{8}$

The correlation between QoL and body mass index was tested using the Pearson test and showed no significant correlation $(\mathrm{p}=0.473)$. Most patients $(46.52 \%)$ had a BMI of $18.5-22.9 \mathrm{~kg} / \mathrm{m}^{2}$ (normoweight). Kalantar-Zadeh et al in 2001 found routine hemodialysis patients with high BMI classifications have lower QoL (measured with SF-36) compared to patients whose BMI lies in the normal range. ${ }^{10}$ The study by Feroze et al (2010) stated that a higher body fat percentage is linked to lower QoL. ${ }^{20}$

The correlation between QoL and HD adequacy measured by $\mathrm{Kt} / \mathrm{V}$ using the Pearson test showed significant correlations $(\mathrm{p}=.082)$. The mean $\mathrm{Kt} / \mathrm{V}$ value of the subjects was 1.21, where dialysis was done twice weekly for 5 hours each session. This is markedly below the recommended $\mathrm{Kt} / \mathrm{V}$ value of $\geq 1.8$ for patients scheduled for twice-weekly hemodialysis session of 5 hours each session. This result is consistent with the study conducted by Ara et al (2013) in Ulin general hospital Banjarmasin were patients on routine twice weekly hemodialysis with $\mathrm{Kt} / \mathrm{V} \leq 1.8 \mathrm{had}$ significantly lower physical health. All subjects of the study by Ara et al were assumed to have developed mental adaptation to hemodialysis, having received routine hemodialysis 3 months prior to data collection. ${ }^{19}$

The HD shift time of hemodialysis showed no significant association with patients' QoL after being tested with the independent sample T-test $(p>0.05)$. Abassi et al stated that the timing of dialysis impact patients' sleeping pattern, especially patients scheduled to have morning dialysis. ${ }^{21}$ However, no association was found between the timing of hemodialysis with QoL. ${ }^{21}$ A study by Norozi et al in 125 patients who underwent routine chronic hemodialysis in 8 teaching hospitals in Iran showed no significant association between timing of HD and patients' QoL. ${ }^{22}$ According to Saad et al, morning dialysis is an independent factor for depression and sleeping disorder. $^{23}$ while Teles et al found that multiple factors affect the sleeping pattern and depression of routine HD patients other than the timing of HD, such as anemia, old age, and low serum phosphate. ${ }^{24}$

\section{CONCLUSION}

No significant correlation was found between QoL and hemoglobin levels, BMI, and timing of HD in patients receiving twice-weekly HD lasting 5 hours per session.
A significant correlation was found between serum albumin, and $\mathrm{Kt} / \mathrm{V}$, with QoL.

The abstract of this original article has previously been displayed in 54th Annual Scientific Meeting of the Australian and New Zealand Society of Nephrology annual scientific meeting 2018 in the form of a poster and online which can be accessed in the Wiley Online Library: https://doi.org/10.1111/ nep. 13442

\section{REFERENCES}

1. Levey A, Coresh J, et al. Clinical practice for chronic kidney disease evaluation classification and stratification. Am J Kidney Dis. 2002 Feb; 39(2):43-6

2. Suhardjono. Hemodialysis; Clinical and basic principal. In: Siti Setiati, Idrus Alwi, Aru Sudoyo, Marcellus Simadibrata, Bambang Setiyadi, Ari Fharial Syam. Internal Medicine Textbook. Publishing Center of the Internal Medicine Department. 2014 July; 4: 2192-95

3. S Abraham, A. Ramachandran. Estimation of Quality of Life in Haemodialysis Patients. Indian Journal of Pharmaceutical Sciences. 2012 Dec; 74(6):583-87.

4. Valderrabano F, Jofre R, et al. Quality of Life in End Stage Renal Disease Patients. Am J Kidney Dis. 2001 Sept; 38(3):443-59

5. Moreno F, Sanz-Guajardo D, et al. Increasing the Hematocrit has a Beneficial Effect on Quality of life and is Safe in Selected Hemodialysis Patients. J Am Soc Nephrol. 2000 July; 11:33542

6. Beusterien K, Nissenson A, et al. The Effects of Recombinant Human Erythropoietin on Functional Health and Well-being in Chronic Dialysis Patients. J Am Soc Nephrol. 1996 Jan;7:763-73

7. A. Cupisti, A. Valeri, et al. Clinical Study: Food Intake and Nutritional Status in Stable Hemodialysis Patients. Informa Healthcare. 2010 Oct;32:47-54

8. Kimmel P, Emont S, et al. ESRD Patient Quality of Life: Symptom, Spiritual Beliefs, Psychosocial Factors, and Ethnicity. Am J Kidney Dis. 2003 Oct;42(4):713-21 
9. Arora P. Chronic Kidney Disease [Internet]. USA: Medscape; 2016 [cited 2016 Jun 25]. Available from: http://emedicine.medscape. com/article/238798-overview

10. Kalantar K, Kopple J, et al. Association Among SF36 Quality of Life Measures and Nutrition, Hospitalization, and Mortality in Hemodialysis. J Am Soc Nephrol. 2001 Jan;12: 2797-06

11. Pernefri. Dialysis Consensus. Indonesian Nephrology Society Publishing. 2003

12. Hecking M, Bieber B, et al. Sex-Specific Differences in Hemodialysis Prevalence and Practice and the Male-to-Female Mortality Rate: The Dialysis Outcomes and Practice Patterns Study (DOPPS). Plos Medicine. 2014 Oct 28;11(10):2-4

13. End-Stage Renal Disease in The United States [Internet]. USA: U.S Renal data system annual data report; 2015 [cited 2016 July 2]. Available from: https://www.kidney.org/news/newsroom/ factsheets/End-Stage-Renal-Disease-in-the-US

14. Rossi E. Red Cell Transfusion Therapy in Chronic Anemia. Transfusion Medicine I. 1994 Jan;6(8):1045-64

15. Hebert P, Hu L, et al. Review of Physiologic Mechanism in Response to Anemia. Canadian Medical Association. 1997 June 1; 156(11):2736

16. Drueke T, Locatelli F, et al. Normalization of Hemoglobin Level in Patients with Chronic Kidney Disease and Anemia. N Engl J Med. 2006 Nov 16;355(20):2078-080

17. Soleymanian T, Nejati M, et al. SF36 Quality of Life and Mortality Across Different Levels of Serum Albumin in Patients With Hemodialysis. Nephrourol Mon.2017 July; 9(4):1-7

18. Kubrusly M, Maria C, Santos D, et al. Comparative Analysis of Pre- and Post-Dialysis Albumin Levels as Indicators of Nutritional and Morbidity and Mortality Risk in Hemodialysis Patients. J Bras Nefrol. 2012 Jan;34(1):27-35

19. Rizky A, Rudianssyah M, et al. Correlation Between Hemodialysis Adequacy and Quality of Life of Patients in Ulin Banjarmasin Hospital. Berkala Kedokteran.2013 Jan; 2(9):151-160

20. Feroze M, Noori N, et al. Quality of Life and Mortality in Hemodialysis Patients: Roles of Race and Nutritional Status. Clin J Am Soc Nephrol. 2011 May; 6: 1100-111
21. Abassi M, Safari A, et al. Sleep Disorders in ESRD Patients Undergoing Hemodialysis. Acta Medica Iranica. 2016 Jan;54(3):179-80

22. Firoz MN, Shafipour V, et al. Evaluation of Subjective Sleep Quality in Hemodialysis Patients and Its Association with Hemodialysis Timing. Iran. JNMS. 2015 Oct 26; 2(4):43-50

23. Saad M, Douaihy Y, et al. Predictors of Quality of Life in Patients with End Stage Renal Disease on Hemodialysis. Int J Nephrol Renovasc Dis. 2015 Sept 3; 8:119-23

24. Teles F, Azevedo V, et al. Depression in Hemodialysis Patients: the Role of Dialysis Shift. Clinics. 2014 Jan;69(3):198-202 